\title{
Relationship between augmented eccentric connectivity index and some other graph invariants
}

\author{
Nilanjan De \\ Department of Basic Sciences and Humanities (Mathematics),Calcutta Institute of Engineering and Management, \\ Kolkata, West Bengal, India. \\ E-mail:de.nilanjan@rediffmail.com
}

\begin{abstract}
The augmented eccentric connectivity index of a graph which is a generalization of eccentric connectivity index is defined as the summation of the quotients of the product of adjacent vertex degrees and eccentricity of the concerned vertex of a graph. In this paper we established some relationships between augmented eccentric connectivity index and several other graph invariants like number of vertices, number of edges, maximum vertex degree, minimum vertex degree, the total eccentricity index the first Zagreb indices and the second multiplicative Zagreb index.
\end{abstract}

Keywords:Vertex Degree, Eccentricity, Zagreb Eccentricity Indices.

\section{Introduction}

In this paper we are concerned only with finite simple connected graph i.e. finite connected graph without self loops and multiple edges. For a graph $G$ let the set of all vertices and edges are denoted by $V(G)$ and $E(G)$ respectively. Also let $n$ and $m$ be, respectively, the number of vertices and edges of $G$. For all $v$ belongs to $V(G) \operatorname{let} \operatorname{deg}(v)$ be the number of first neighbor i.e. degree of the vertex $v$. Also let $\Delta=\max \{\operatorname{deg}(v): v \in V(G)\}$ and $\delta=\min \{\operatorname{deg}(v): v \in V(G)\}$ be the maximum and the minimum vertex degree, respectively. Let the distance between any two vertices of $V(G)$, is equal to the length of the shortest path connecting them and denoted by $d(u, v)$. Also for a given vertex of $V(G)$ its eccentricity is the largest distance from that vertex to any other vertices of $G$

$$
\text { i.e. } \varepsilon(v)=\max \{d(v, x): x \in V(G)\}
$$

The radius and diameter of the graph are the smallest and largest eccentricity among all the vertices of $G$ respectively [1].

$$
\text { i.e. } r=r(G)=\min \{\varepsilon(v): v \in V(G)\} \text { and } d=d(G)=\max \{\varepsilon(v): v \in V(G)\}
$$

Also the total eccentricity of a graph, denoted by $\theta(G)$, is the sum of eccentricities of all the vertices of $G$ [1].

The oldest and most popular graph invariant, the classical Zagreb indices, introduced by Gutman and Trinajstić are defined as [15]

$$
M_{1}(G)=\sum_{v \in V(G)} \operatorname{deg}(v)^{2} \quad, \quad M_{2}(G)=\sum_{(u, v) \in E(G)} \operatorname{deg}(u) \operatorname{deg}(v)
$$

where $\operatorname{deg}(v)$ is the degree of the vertex $v$. The Zagreb indices were subject to a large number of mathematical studies [14].

Todeschini et al. [16] have introduced the multiplicative variants of additive graph invariants, which applied to the Zagreb indices would lead to the first and second Multiplicative Zagreb Index which are defined as

$$
\prod_{1}(G)=\prod_{v \in V(G)} \operatorname{deg}(v)^{2}, \quad \prod_{2}(G)=\prod_{(u, v) \in E(G)} \operatorname{deg}(u) \operatorname{deg}(v)
$$


The properties of these multiplicative Zagreb indices for trees were studied by Gutman [10] and some properties, bounds were studied by Liu and Zhang [10].

In recent years a number of graph invariants related to eccentricity have been derived and studied. One of them the eccentric connectivity index of a graph G was proposed by Sharma, Goswami and Madan [12] and is defined as

$$
\xi^{a c}(G)=\sum_{v \in V(G)} \operatorname{deg}(v) \varepsilon(v)
$$

A more generalization of the connective eccentric index known as augmented eccentric connectivity index was introduced by Gupta, Singh and Madan [8] and is defined as

$$
\xi^{a c}(G)=\sum_{v \in V(G)} \frac{M(v)}{\varepsilon(v)}
$$

where $M(v)$ denotes the product of degrees of all neighbors of vertex $v_{\mathrm{i}}$ i.e. $M(v)=\prod_{(u, v) \in E(G)} \operatorname{deg}(u)$. This index is relatively new though subject to some chemical as well as mathematical studies. From above definition of augmented eccentric connectivity index it is evident that, as the degrees are taken over the neighborhoods and then multiplied and again the reciprocal of eccentricity is considered for a vertex, so the contribution of a vertex to this index is both nonlocal and non-linear.Different properties of augmented eccentric connectivity index have been studied by Došlić and Saheli (2011)[6], Sedlar (2012)[11], De (2012)[3].

The problem of finding extremal properties of some topological indices so that to establish some inequalities involving different graph invariants have been studied by different researchers of which only some recent results are mentioned here $[2,4,5,10,17]$. The aim of this paper is to investigate similar extremal properties for multiplicative augmented eccentric connectivity index. In this paper we first establish some upper and lower bounds of $\sum_{v \in V(G)} M(v)$ and then using those some sharp lower and upper bounds of augmented eccentric connectivity index is given in terms of different graph invariants including the number of vertices $(n)$, number of edges $(m)$, radius $(r)$, diameter $(d)$, maximum vertex degree $(\Delta)$, minimum vertex degree $(\delta)$, the total eccentricity index $(\theta(G))$ the first Zagreb indices $\left(\mathrm{M}_{1}(\mathrm{G})\right)$ and the second multiplicative Zagreb index $\left(\Pi_{2}(G)\right)$.

\section{Main results}

The First we find some upper and lower bounds of summation of degrees of all the neighbors of the vertices of $G$ is $\sum_{v \in V(G)} M(v)$.

Proposition 2.1: Let $G$ be a simple connected graph with $n$ vertices and $\Pi_{1}(G)$ denotes the first Zagreb index of $G$, then

$$
M(v) \geq n \Pi_{2}(G)^{\frac{1}{n}}
$$

with equality if and only if all the vertices of $G$ are of same degree.

Proof. Using inequality between arithmetic and geometric mean, we have

$$
\begin{gathered}
\frac{1}{n} \sum_{v \in V(G)} M(v) \geq\left[\prod_{v \in V(G)} M(v)\right]^{\frac{1}{n}}=\left[\prod_{v \in V(G)} \prod_{(u, v) \in E(G)} \operatorname{deg}(v)\right]^{\frac{1}{n}} \\
\quad \text { i.e. } \sum_{v \in V(G)} M(v) \geq n\left[\prod_{v \in V(G)} \operatorname{deg}(v)^{\operatorname{deg}(v)}\right] \geq n\left[\Pi_{2}(G)\right]^{\frac{1}{n}}
\end{gathered}
$$

with equality if and only if all the vertices of $G$ are of same degree. Here $\Pi_{2}(G)$ is the second multiplicative Zagreb index of $\mathrm{G}$ and is defined as [9] 


$$
\Pi_{2}(G)=\prod_{(u, v) \in E(G)} \operatorname{deg}(u) \operatorname{deg}(v)=\prod_{v \in V(G)} \operatorname{deg}(v)^{\operatorname{deg}(v)} .
$$

Proposition 2.2: Let $G$ be a simple connected graph with $n$ vertices and $M_{1}(G)$ denotes the first Zagreb index of $G$, then

with equality if and only if $G \cong K_{2}$.

$$
\sum_{v \in V(G)} M(v) \geq M_{1}(G)
$$

Proof. Let $\delta(v)$ denotes the sum of degrees of all the neighbors of the vertex $v$, so that

$$
\delta(v)=\sum_{(u, v) \in E(G)} \operatorname{deg}(u)
$$

So obviously from the definition of $M(v)$

$$
M(v) \geq \delta(v) \text { for all } v \in V(G)
$$

Thus, $\sum_{v \in V(G)} M(v) \geq \sum_{v \in V(G)} \delta(v)=M_{1}(G)$

with equality if and only if $\operatorname{deg}(v)=1$ for all $v \in V(G)$. Here $M_{1}(G)$ is the first Zagreb eccentricity index of $G$ and is defined as [14]

$$
M_{1}(G)=\sum_{v \in V(G)} \operatorname{deg}(v)^{2}=\sum_{v \in V(G)} \delta(v)
$$

Proposition 2.3: Let $G$ be a simple connected graph with $n$ vertices, then

$$
\sum_{v \in V(G)} M(v) \leq n\left(\frac{\Delta^{2}}{\delta}\right)^{\Delta}
$$

with equality if and only if all the vertices of $G$ are of same degree.

Proof. Using the inequality between arithmetic and geometric mean, we have

$$
\frac{1}{\operatorname{deg}(v)} \sum_{(u, v) \in E(G)} \operatorname{deg}(u) \geq\left[\prod_{(u, v) \in E(G)} \operatorname{deg}(u)\right]^{\frac{1}{\operatorname{deg}(v)}}
$$

i.e. $M(v) \leq\left[\frac{\delta(v)}{\operatorname{deg}(v)}\right]^{\operatorname{deg}(v)}$

Now since, $\delta \leq \operatorname{deg}(v) \leq \Delta$, so

$$
\delta(v)=\sum_{(u, v) \in E(G)} \operatorname{deg}(u) \leq \Delta^{2}
$$

Thus from (2.1), we can write

$$
M(v) \leq\left[\frac{\Delta^{2}}{\delta}\right]^{\Delta}
$$

from where the desired result follows with equality if and only if all the vertices of $G$ are of same degree.

Proposition 2.4: Let $G$ be a simple connected graph with $n$ vertices, then

$$
\sum_{v \in V(G)} M(v) \leq n\left(\frac{2 m}{\delta}+\delta-n\right)^{\Delta}
$$

with equality if and only if all the vertices of $G$ are of same degree. 
Proof. We have [14]

for all $v \in V(G)$, so that

$$
\delta(v) \leq 2 m-\operatorname{deg}(v)-\delta(n-1-\operatorname{deg}(v))
$$

$$
\frac{\delta(v)}{\operatorname{deg}(v)} \leq \frac{2 m}{\operatorname{deg}(v)}-1-\frac{\delta(n-1)}{\operatorname{deg}(v)}+\delta \leq \frac{2 m}{\delta}-n+\delta
$$

Thus, $\left[\frac{\delta(v)}{\operatorname{deg}(v)}\right]^{\operatorname{deg}(v)} \leq\left[\frac{2 m}{\delta}-n+\delta\right]^{\Lambda}$.

So from (1) we have,

$$
\sum_{v \in V(G)} M(v) \leq \sum_{v \in V(G)}\left[\frac{\delta(v)}{\operatorname{deg}(v)}\right]^{\operatorname{deg}(v)} \leq n\left(\frac{2 m}{\delta}+\delta-n\right)^{\Delta}
$$

which is our desired result. In the above inequality equality holds if and only if all the vertices of $G$ are of same degree.

Now we find some upper and lower bounds of augmented eccentric connectivity index.

Theorem 2.1: Let $G$ be a simple connected graph with $n$ vertices and $m$ edges, then

$$
\frac{\delta^{\delta}}{d} \leq \frac{1}{n} \xi^{a c}(G) \leq \frac{\Delta^{\Delta}}{r}
$$

with equality if and only if $G$ all the vertices of $G$ are of same degree and eccentricity.

Proof. Since $\delta \leq \operatorname{deg}(v) \leq \Delta$, for all $v \in V(G)$ and $M(v)$ is the product of all the neighbors of the vertex $v$, we have $\delta^{\delta} \leq M(v) \leq \Delta^{\Delta}$, for all $v \in V(G)$, with equality if and only if $G$ is a regular graph. Similarly since $r \leq \varepsilon(v) \leq d$, for all $v \in V(G)$, from the definition of augmented eccentric connectivity index, we have

$$
\frac{n \delta^{\delta}}{d} \leq \xi^{a c}(G) \leq \frac{n \Delta^{\Delta}}{r}
$$

which is our desired result. Obviously in the above inequality equality holds if and only if all the vertices are of same degree and eccentricity.

Theorem 2.2: Let $G$ be a simple connected graph with $n$ vertices and $m$ edges, then

$$
\xi^{a c}(G) \geq \frac{n^{2} \Pi_{2}(G)^{\frac{1}{n}}}{\theta(G)}
$$

with equality if and only if all the vertices of $G$ are of same degree and eccentricity.

Proof. To prove this theorem we use Chebyschev's inequality as follows

$$
n \xi^{a c}(G)=n \sum_{v \in V(G)} \frac{M(v)}{\varepsilon(v)} \geq \sum_{v \in V(G)} M(v) \sum_{v \in V(G)} \frac{1}{\varepsilon(v)}
$$

with equality if and only if all the vertices of $G$ are same degree and eccentricity. Now using the inequality between arithmetic and harmonic mean, we have 


$$
\begin{aligned}
& \frac{1}{n} \sum_{v \in V(G)} \varepsilon(v) \geq \frac{n}{\sum_{v \in V(G)} \varepsilon(v)} \\
& \text { i.e. } \sum_{v \in V(G)} \frac{1}{\varepsilon(v)} \geq \frac{n^{2}}{\theta(G)}
\end{aligned}
$$

with equality if and only if all the vertices of $G$ are of same eccentricity. So from above we can write

$$
\xi^{a c}(G) \geq \frac{n}{\theta(G)} \sum_{v \in V(G)} M(v)
$$

Now using Proposition 2.1 from above the desired result follows, with equality if and only if all the vertices of $G$ are of same degree and eccentricity.

Corollary 2.1: Let $G$ be a simple connected graph with $n$ vertices and $m$ edges, then

$$
\xi^{a c}(G) \geq n \frac{M_{1}(G)}{\theta(G)}
$$

where $M_{1}(G)$ is the first Zagreb index of $G$. In the above inequality, equality holds if and only if $G$ is a path of length one.

Proof. Using proposition 2.2, the above result follows from relation (2.2). Obviously equality holds in the above inequality if and only if $G \cong K_{2}$.

Theorem 2.3: Let $G$ be a simple connected graph, then

$$
\xi^{a c}(G) \leq \frac{n}{n-\delta}\left(\frac{\Delta^{2}}{\delta}\right)^{\Delta}
$$

with equality if and only if $G \cong K_{n}$.

Proof. Since we have [13]

$$
\frac{1}{\varepsilon(v)} \leq \frac{1}{n-\delta}
$$

for all $v \in V(G)$, with equality if and only if $G \cong K_{n}-j e$ for $j=1,2, \ldots .,\lfloor n / 2\rfloor$ or $G \cong P_{4}$, so from the definition of augmented eccentric connectivity index, we can write

$$
\xi^{a c}(G) \geq \frac{1}{n-\delta} \sum_{v \in V(G)} M(v)
$$

Now using Proposition 2.3, the desired result follows from (2.3). The equality holds in the Proposition 2.3 if all the vertices are of same degree. Thus in this theorem equality holds if and only if $G \cong K_{n}$.

Corollary 2.2: Let $G$ be a simple connected graph with $n$ vertices and $m$ edges, then

$$
\xi^{a c}(G) \leq \frac{n}{(n-\delta)}\left(\frac{2 m}{\delta}+\delta-n\right)^{\Delta}
$$

with equality if and only if $G \cong K_{n}$.

Proof. Using Proposition 2.4, the above result follows from relation (2.3). The equality holds in the Proposition 2.4 if and only if all the vertices are of same degree. Thus in this result equality holds if and only if $G \cong K_{n}$. 
Theorem 2.4: Let G be a simple connected graph, then

$$
\xi^{a c}(G) \leq \frac{n(n-1) \Delta^{2 \Delta}}{\delta^{\Delta+1}}
$$

with equality if and only if $G \cong K_{n}$.

Proof. Since we have [13]

$$
\frac{1}{\varepsilon(v)} \leq \frac{n-1}{D(v)}
$$

for all $v \in V(G)$, with equality if and only if $G \cong K_{n}$ so

$$
\sum_{v \in V(G)} \frac{1}{\varepsilon(v)} \leq(n-1) \sum_{v \in V(G)} \frac{1}{D(v)}
$$

Now since, $D(v) \geq \operatorname{deg}(v)$ for all $v \in V(G)$, with equality if and only if $G \cong K_{n}$, we have from above

$$
\sum_{v \in V(G)} \frac{1}{\varepsilon(v)} \leq(n-1) \sum_{v \in V(G)} \frac{1}{\operatorname{deg}(v)}
$$

Again since, $D(v) \geq \operatorname{deg}(v)$ for all $v \in V(G)$, we can write

$$
\sum_{v \in V(G)} \frac{1}{\varepsilon(v)} \leq \frac{n(n-1)}{\delta}
$$

So, using definition of augmented eccentric connectivity index and Proposition 2.3, the desired result follows from (2.4). Obviously in the above inequality equality holds if and only if $G \cong K_{n}$.

Corollary 2.3: Let $G$ be a simple connected graph with $n$ vertices and $m$ edges, then

$$
\xi^{a c}(G) \leq \frac{n(n-1)}{\delta}\left(\frac{2 m}{\delta}+\delta-n\right)^{\Delta}
$$

with equality if and only if $G \cong K_{n}$.

Proof. From Proposition 2.4, we have

$$
M(v) \leq\left(\frac{2 m}{\delta}+\delta-n\right)^{\Delta}
$$

with equality if and only if $G$ is regular. So from the definition of augmented eccentric connectivity index we can write

$$
\xi^{a c}(G) \leq\left(\frac{2 m}{\delta}+\delta-n\right)^{\Delta} \sum_{v \in V(G)} \frac{1}{\varepsilon(v)}
$$

Now using (2.4), from above we get the desired result. Since the equality holds in the Proposition 2.4 if and only if all the vertices are of same degree. Thus in this result equality holds if and only if $G \cong K_{n}$.

\section{Conclusion}

In this paper, we have established sharp upper and lower bounds foraugmented eccentric connectivity index in terms of graphparameters including the order, size, radius, diameter, maximum vertex degree, minimum vertex degree, the total 
eccentricity index, the first Zagreb indicesand the second multiplicative Zagreb index, so that different inequalities related to augmented eccentric connectivity index to some other graph invariants with the respective equality condition were established.It may be interesting to give the bounds for augmented index in terms of other graph invariants.

\section{References}

[1] P.Dankelmann, W.Goddard, Swart C S (2004). “The average eccentricity of a graph and its subgraphs”, Util. Math., 65, pp.41-51.

[2] N. De (2012). "Some bounds of reformulated Zagreb indices", Applied Mathematical Sciences, 6,pp.5005-5012.

[3] N. De (2012). “Augmented Eccentric Connectivity Index of Some Thorn Graphs”. International Journal of Applied Mathematical Research, 1(4),pp.671-680.

[4] N. De (2012). "Bounds for the connective eccentric index", International Journal of Contemporary Mathematical Sciences, 7(44): pp.21612166.

[5] N. De (2013), "New Bounds for Zagreb Eccentricity Indices", Open Journal of Discrete Mathematics. ,3(1), pp. 70-74.

[6] T.Došlić, M. Saheli (2011). “Augmented Eccentric Connectivity Index”, Miskolc Mathematical Notes, 12(2),pp.149-157.

[7] H. Dureja, A. K.Madan (2007). "Superaugmented eccentric connectivity indices: new generation highly discriminating topological descriptors for QSAR/QSPR modeling”, Med. Chem. Res., 16,pp.331-341.

[8] S.Gupta, M. Singh, A. K. Madan (2000). "Connective eccentricity Index: A novel topological descriptor for predicting biological activity", Journal of Molecular Graphics and Modelling, 18,pp.18-25.

[9] I. Gutman (2011). "Multiplicative Zagreb Indices of Trees", Bulletin of Society of .Mathematics Banja Luka,18,pp.17-23.

[10] J. Liu, V. Zhang(2012).”Sharp Upper Bounds for Multiplicative Zagreb Indices”, MATCH Communications in Mathematical and in Computer Chemistry, 68: pp.231-240.

[11] J. Sedlar (2012). "On augmented eccentric connectivity index of graphs and trees", MATCH Communications in Mathematical and in Computer Chemistry, 68, pp.325-342.

[12] V. Sharma, R. Goswami, A. K. Madan (1997). "Eccentric connectivity index: A novel highly discriminating topological descriptor for structure-property and structure-activity studies", Chemical Information Modeling, 37, pp.273-282.

[13] B. Zhou, Z. Du, "On eccentric connectivity index", MATCH Commun. Math. Comput. Chem., 63 (2010),pp.181-198.

[14] K. C. Das, I. Gutman, "Some properties of the second Zagreb index", MATCH Commun. Math. Comput. Chem., 52 (2004),pp.103-112.

[15] I. Gutman, N. Trinajstić, Graph theory and molecular orbitals, Total $\pi$-electron energy of alternant hydrocarbons, Chem. Phys. Lett., 17 (1972), 535-538.

[16] R. Todeschini, V. Consonni, New local vertex invariants and molecular descriptors based on functions of the vertex degrees, MATCHCommun. Math. Comput. Chem. 64 (2010) 359-372.

[17] N. De, "On multiplicative Zagreb eccentricity indices", South Asian J Math, 2012, 2(6), pp.570-577. 